\title{
Reorientation and coupling effects in polarized heavy ion fusion
}

\author{
I. Martel, J. Gómez-Camacho and M.V. Andrés \\ Departamento de Fisica Atómica, Molecular y Nuclear, Facultad de Física, Apdo. 1065, E-41080 Sevilla, Spain
}

Received 22 October 1991; revised manuscript received 28 January 1992

\begin{abstract}
A simple model to understand the reaction mechanisms in the fusion of polarized heavy ions based on tidal symmetry, the sudden approximation and barrier penetration is presented. The model is applied to ${ }^{23} \mathrm{Na}+{ }^{208} \mathrm{~Pb}$. The effect of the ground state reorientation and the coupling to rotational states is studied. Enhancement of the sub-barrier fusion cross sections and the fusion $J$-moments just above the barrier are found. The polarization of the projectile affects strongly the fusion around and below the Coulomb barrier.
\end{abstract}

During recent years, detailed experimental and theoretical investigations have been carried out on heavy ion fusion at energies close to the Coulomb barrier (sce ref. [1] for a recent revicw). At these encrgies, the interplay between the relative motion and the internal degrees of frecdom is essential, leading to different phenomena such as the sub-barrier fusion enhancement and the increase of the $J$-moments $[2,3]$. Also, the fusion induced by polarized ${ }^{23} \mathrm{Na}$ shows large analyzing powers below the Coulomb barricr [4]. Although several descriptions are used to investigate theoretically the fusion [5], the coupled channels approach has been generally successful to reproduce the experimental data. However, it has problems [6] associated to the use of phenomenological imaginary potentials, the intrinsic complexity of the calculations, and the long computing time required, especially in the situations where the Coulomb force becomes important. Besides, although they give the effect of the coupling to each individual state on the observables, it is not easy to extract from them a clear interpretation of the aspects of the structure and the reaction mechanisms which are most significant for fusion. So, coupled channels calculations necd to be supplemented with simpler calculations containing the important features of the coupled channcls that exhibit their effects on the physical observables.

The objective of this work is to study the effect of reorientation and coupling to rotational states on the fusion cross sections and $J$-distributions induced by the collision of a polarized deformed nucleus with a spherical one. We apply the model to the system ${ }^{23} \mathrm{Na}+{ }^{208} \mathrm{~Pb}$ at energies around the Coulomb barricr. Some of our results can be contrasted with the coupled channels calculations and experimental data [4] for ${ }^{23} \mathrm{Na}+{ }^{206} \mathrm{~Pb}$. Wc take the following approximations:

Tidal symmetry [7]. It arises when the interaction between two particles is invariant with respect to rotations of the interacting particles about their relative coordinate. If the difference in centrifugal barriers of the channels coupled together is ignored, the projection of the spin over the relative coordinate (tidal spin) is conserved and the coupled channel equations can be partially diagonalized. The application of tidal symmetry to fusion [8] leads to the following expression for the partial fusion cross section of a projectile of spin $I$ and projection $M$ along the bcam direction leading to a compound nucleus of $\operatorname{spin} J$ :

$\sigma_{I M}^{J}=\sum_{N^{\prime}}\left|d_{M N}^{I}\left(\pi / 2+\theta_{J} / 2\right)\right|^{2} \sigma_{I}^{J N}$,

where $\sigma_{I}^{J N}$ is the partial fusion cross section for a tidal spin $N$, and $\theta_{J}$ is the classical scattering angle corresponding to an angular momentum $J$. This expression means that the fusion for an angular momentum $J$ occurs as if the projection of the spin along the bisecting direction of the classical trajectory corresponding to $J$ was conserved (cf. ref. [8]). For ener- 
gies close to the barrier, the Coulomb force dominates and $\tan \left(\theta_{J} / 2\right)=\eta / \sqrt{J(J+1)}$ where $\eta$ is the Sommerfeld parameter. The partial fusion cross sections $\sigma_{I}^{J N}$ can be obtained from a reduced coupled channels calculation involving only spin-0 states $[7,8]$. Instead, we will use the sudden approximation.

Sudden approximation [9]. If the excitation energies of the states coupled together are ignored, and all the form factors have the samc geomctry, the reduced coupled channels system can be fully diagonalized, and the partial fusion cross sections can be written as

$\sigma_{i}^{J N}=\sum_{\phi} \omega_{Y \phi}^{N} \sigma^{J N \phi}$

where $\phi$ stands for the eigenchannel of the coupled channel system, $\omega_{I \phi}^{N}$ is the square of the overlap of the ground state $I$ with the eigenchannel $\phi$ for a tidal spin $N$ and $\sigma^{N Q}$ is the partial fusion cross section for the eigenchannel $\phi$. This channel is characterized by a diagonal monopole potential $V_{\phi N}(r)$. $\sigma^{J N_{\phi}}$ could bc calculated by an optical model calculation, using a short-range imaginary potential or incoming boundary conditions. Instead, we will use barrier penetration.

Barrier penetration. In order to avoid the introduction of imaginary potentials, we will calculate the partial fusion cross sections for the cigenchannels from the barrier penetration probability. Thus,

$\sigma^{J N \phi}(E)=\frac{\pi \hbar^{2}(2 J+1)}{2 \mu E} T(J, E)$.

Using the comparison method [10], which is a generalization of the WKB method for two turning points, the probability of penetration can be written as $T(J, E)=\{1+\exp [\varepsilon(J, E)]\}^{-1}$ where

$\varepsilon(J, E)=2 \int_{k_{1}}^{R_{2}}\left(\frac{2 \mu}{\hbar^{2}}\left[V_{\phi N}(r)-E\right]+\frac{J(J+1)}{r^{2}}\right)^{1 / 2} \mathrm{~d} r$.

$R_{1}$ and $R_{2}$ are the classical turning points. Note that this expression is valid both below and above the barricr. In the latter case, the turning points are complex, and the integral is performed in the complex plane. In this approach, whenever the interacting nuclei pass the barrier, they go to fusion channels. This is equivalent to using incoming boundary conditions or an optical potential with a deep, short-range imaginary part.

We apply this model to the system ${ }^{23} \mathrm{Na}+{ }^{208} \mathrm{~Pb}$. The target ${ }^{208} \mathrm{~Pb}$ is considered as a spherical, inert nucleus of radius $R_{\mathrm{Pb}}=1.2 A^{1 / 3} \mathrm{fm}$. The states of the projectile ${ }^{23} \mathrm{Na}$ form a rotational band with $K=\frac{3}{2}$. The intrinsic shape of the projectile is characterized by a quadrupole deformation,

$R_{\mathrm{Na}}(\theta)=R_{\mathrm{Na}}^{0}\left(1+\sqrt{\frac{5}{4 \pi}} \beta_{2} P_{2}(\cos \theta)\right)$,

where $R_{\mathrm{Na}}^{0}=1.2 A^{1 / 3} \mathrm{fm}$ and the deformation parameter $\beta_{2}=0.441$ is obtained (to all orders in $\beta_{2}$ ) from the intrinsic quadrupole moment of ${ }^{23} \mathrm{Na}, Q_{i}=50.3$ $\mathrm{fm}^{2}$, derived in the rotational model from the spectroscopic quadrupole moment of ${ }^{23} \mathrm{Na}, Q=10.06(20)$ $\mathrm{fm}^{2}[11]$.

The nuclear potential is given by a deformed proximity potential [12],

$$
\begin{aligned}
& V_{\text {Nucl }}(r, \theta)=-S \frac{R_{\mathrm{Na}}^{0} R_{\mathrm{Pb}}}{R_{\mathrm{Na}}^{0}+R_{\mathrm{Pb}}}\left[1-B_{2} P_{2}(\cos \theta)\right] \\
& \quad \times \exp \left(\frac{R_{\mathrm{Na}}(\theta)+R_{\mathrm{Pb}}-r}{a}\right), \\
& B_{2}=\frac{2 R_{\mathrm{Pb}}}{R_{\mathrm{Na}}^{0}+R_{\mathrm{Pb}}} \sqrt{\frac{5}{4 \pi}} \beta_{2},
\end{aligned}
$$

where $\theta$ is the angle between the projectile symmetry axis and the relative coordinate, $S=50 \mathrm{MeV} / \mathrm{fm}$ and $a=0.63 \mathrm{fm}$. From this expression, we retain the monopole and quadrupole form factors. Thus

$$
\begin{aligned}
& V_{\text {Nucl }}(r, \theta)=-\exp \left(\frac{R_{\mathrm{Na}}^{0}+R_{\mathrm{Pb}}-r}{a}\right) \\
& \quad \times\left[V_{0}+V_{2} P_{2}(\cos \theta)\right]
\end{aligned}
$$

where $V_{0}=129 \mathrm{MeV}$ and $V_{2}=155 \mathrm{McV}$. The Coulomb potential has monopole and quadrupole terms:

$$
V_{\text {Coul }}(r, \theta)=\frac{Z_{1} Z_{2} e^{2}}{r}+\frac{Q_{i} Z_{2} e^{2}}{2 r^{3}} P_{2}(\cos \theta) \text {, }
$$

where $Q_{i}$ is the intrinsic quadrupole moment of the projectile. The solid lines in fig. 1 show the potential for different orientations of the projectile with respect to the relative coordinate.

For the purpose of this paper, we perform three calculations: 


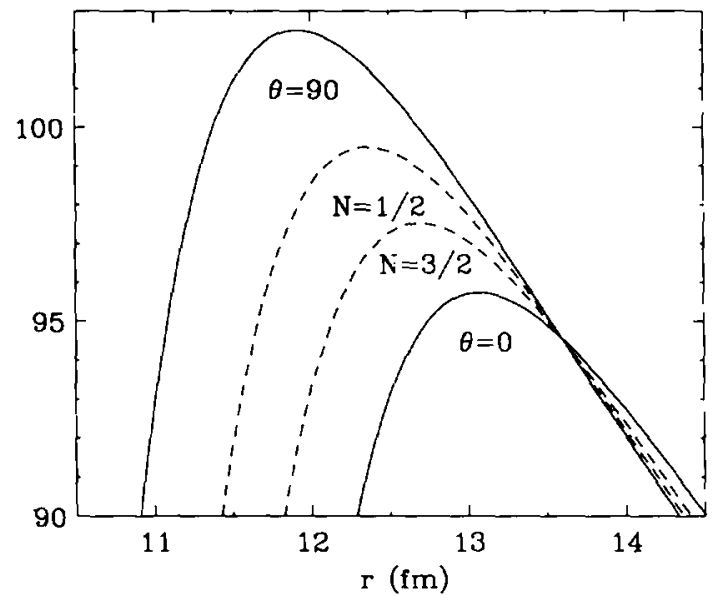

Fig. 1. Nuclear barrier for the system ${ }^{23} \mathrm{Na}+{ }^{208} \mathrm{~Pb}$. The full lines represent the potential $V(r, \theta)=V_{\text {Nucl }}(r, \theta)+V_{\text {Coul }}(r, \theta)$ for $\theta=$ $0^{\circ}$ and $90^{\circ}$. Dashed lines represent the potential $V_{I N}(r)$ for $N=\frac{1}{2}$ and $\frac{3}{2}$ tidal spin values.

(i) Monopole. A one-channel calculation including only the monopole potential.

(ii) Reorientation. Only the ground state reorientation is included. This case corresponds to a rotor with a small moment of inertia, so that excited states are very high in energy, and the coupling to them is unimportant. Classically, the rotor would oscillate very fast in the presence of the non-central forces, so that the relative motion would be affected by an average potential given by (10). The partial fusion cross section $\sigma_{l}^{J N}$ is calculated from the diagonal potential $V_{I N}(r)$, given by

$$
\begin{aligned}
& V_{I N}(r)=\frac{1}{4}(2 I+1) \\
& \times \int_{0}^{\pi} \mathrm{d} \theta \operatorname{sen}(\theta)\left(\left|d_{N K}^{l}(\theta)\right|^{2}+\left|d_{N-K}^{l}(\theta)\right|^{2}\right) V(r, \theta) .
\end{aligned}
$$

The dashed lines in fig. 1 show the potential $V_{I N}(r)$ for tidal spin values $N=\frac{1}{2}$ and $\frac{3}{2}$. The barricr for $N=\frac{1}{2}$ is higher than for $N=\frac{3}{2}$, and occurs at smaller distances.

(iii) Coupling. The whole rotational band is included in the calculation ignoring the excitation energies. This case corresponds to a rotor with a large moment of inertia. Classically, the rotor orientation is fixed during the reaction process. The eigenstates $\phi$ correspond to definite orientations of the rotor with respect to the relative coordinate, characterized by the angle $\theta$. Thus, the sum over $\phi$ in eq. (2) becomes an integral over $\theta$, and the partial fusion cross section is given by

$$
\begin{aligned}
\sigma_{I}^{J N} & =\frac{1}{4}(2 I+1) \\
\times & \int_{0}^{\pi} \mathrm{d} \theta \operatorname{sen}(\theta)\left(\left|d_{N K}^{X}(\theta)\right|^{2}+\left|d_{N-K}^{l}(\theta)\right|^{2}\right) \sigma^{J}(\theta),
\end{aligned}
$$

where $\sigma^{J}(\theta)$ is the partial fusion cross section obtained from the potential $V(r, \theta)$.

The case of ${ }^{23} \mathrm{Na}$ corresponds to an intermediate situation between the reorientation and coupling cases. The states most relevant for the coupling ( $\frac{5}{2}+$ $0.44 \mathrm{MeV}, \frac{7}{2}+2.08 \mathrm{MeV}$ ) have energies comparable to the barrier splitting.

The comparison of these calculation exhibits the relative importance of the "static" aspect of the deformation, dependent on the deformation parameter and associated to the ground state reorientation, and its "dynamic" aspect, dependent on the moment of inertia and associated to the coupling to excited rotational states. Thus, when the reorientation and coupling calculations differ very much for an observable, "dynamic" effects are important. On the contrary, when reorientation and coupling calculations are very similar, but different from the monopole calculation, "static" effects are dominant. In this case the experimental data will give information about the deformation of the potential that is not obscured by the reaction mechanism. Finally, when the three calculations are very similar, the deformation is not important for that magnitude.

Fig. 2 shows the partial fusion cross section $\sigma_{I M}^{J}$ versus $J$ obtained from the reorientation and coupling calculations at different energies. For $E_{\mathrm{Lab}}=100$ $\mathrm{MeV}$ (fig. 2a) the system is below the Coulomb barrier. The fusion for $M=\frac{3}{2}$ is much larger than for $M=\frac{1}{2}$, producing a strong polarization dependence. The coupling calculations give larger fusion cross sections than the reorientation calculations for each $M$ and for all the relevant $J$ values. These results agree qualitatively with the coupled channels calculations presented in ref. [4]. For $E_{\mathrm{l} . \mathrm{b}}=110 \mathrm{MeV}$ the system is roughly at the Coulomb barrier (fig. $2 b$ ), the effect of the coupling is to decrease the partial fusion cross 


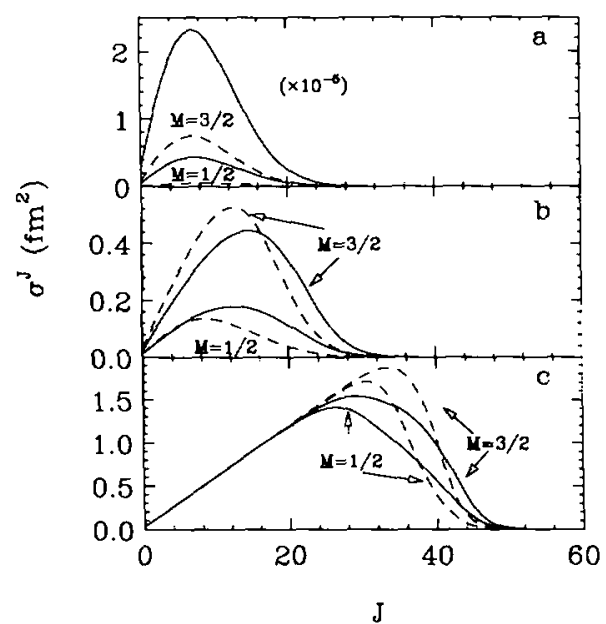

Fig. 2. Partial fusion cross section $\sigma_{I M}^{\prime}$ for ${ }^{23} \mathrm{Na}+{ }^{208} \mathrm{pb}$ at $E_{\text {Lab }}=100 \mathrm{MeV}$ (a), $110 \mathrm{MeV}$ (b) and $120 \mathrm{MeV}$ (c) as a function of $J$. The full lines are the calculations for the adiabatic coupling to all the rotational states, and the dashed lines are the calculations considering only the ground state reorientation, for $M=\frac{1}{2}$ and $\frac{3}{2}$ spin projections.

sections for partial waves below the grazing, and to increase them for high partial waves. This is duc to the fact that, for low partial waves, the system is above the effective barrier for that $J$ value, and the coupled channels effects decrease the cross sections. For high partial waves the system is above the effective barrier and the effect is reversed. For $E_{\mathrm{Lab}}=120 \mathrm{McV}$ the system is above the Coulomb barrier (fig. $2 \mathrm{c}$ ), the fusion cross sections for low partial waves are independent of the polarization. There is a small range of angular momentum around the grazing angular momentum for which there is polarization dependence that is widened by the coupling.

The total fusion cross section and the average value of $J(J+1)$ can be calculated for each value of $M$ using the expressions

$\sigma_{M}=\sum_{J} \sigma_{I M}^{J}$

$\langle J(J+1)\rangle_{M}=\frac{\sum_{J} J(J+1) \sigma_{M M}^{\prime}}{\sigma_{M}}$.

From these values the following observables can be defined:

$\sigma=\left(\sigma_{3 / 2}+\sigma_{1 / 2}\right) / 2$,

$$
\begin{aligned}
& \langle J(J+1)\rangle \\
& =\frac{\langle J(J+1)\rangle_{3 / 2} \sigma_{3 / 2}+\langle J(J+1)\rangle_{1 / 2} \sigma_{1 / 2}}{\sigma_{3 / 2}+\sigma_{1 / 2}}, \\
& T_{20}(\sigma)=\frac{\sigma_{3 / 2}-\sigma_{1 / 2}}{\sigma_{3 / 2}+\sigma_{1 / 2}}, \\
& T_{20}(J(J+1)) \\
& =\frac{\langle J(J+1)\rangle_{3 / 2}-\langle J(J+1)\rangle_{1 / 2}}{\langle J(J+1)\rangle_{3 / 2}+\langle J(J+1)\rangle_{1 / 2}} .
\end{aligned}
$$

Fig. 3 shows the bchavior of these observables versus the scattering energy. Both reoricntation and coupling increase the fusion cross sections (fig. $3 \mathrm{a}$ ) below the barrier and decrease it above the barrier, but the effect of the coupling is much more important. This is in agreement with the coupled channels results [4], and can be understood as an effect of the splitting of the barrier produced by the coupling. They also increase the $J$-moment $\langle J(J+1)\rangle^{1 / 2}$ (fig. 3b) at energies just above the Coulomb barrier. This effect is also due to the splitting of the barriers, which increases the cross sections for large $J$ and decreases those for low $J$, as it was seen in fig. $2 \mathrm{~b}$. Below the

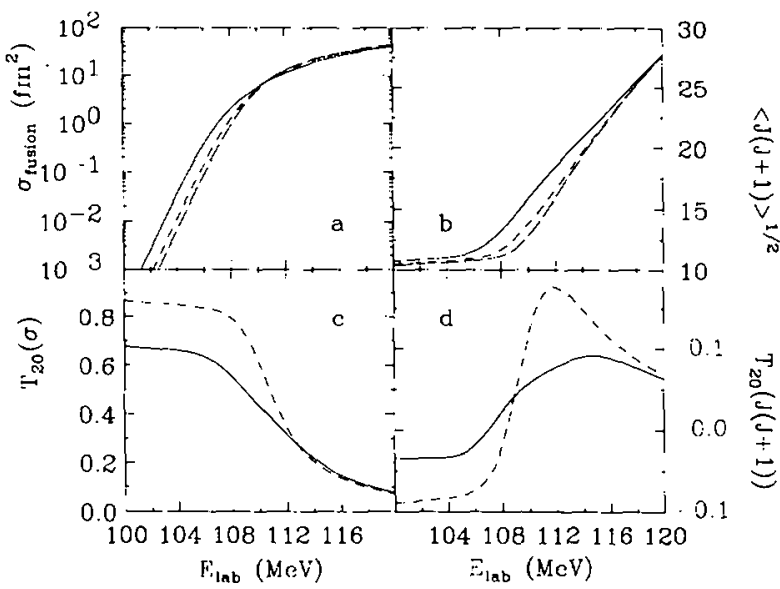

Fig. 3. Total fusion cross section $\sigma_{I}$ (a), $J$-moment $\langle J(J+1)\rangle^{1 / 2}(\mathrm{~b})$, analyzing power $T_{20}(\sigma)(\mathrm{c})$ and $T_{20}(J(J+1))$ (d) for ${ }^{23} \mathrm{Na}+{ }^{208} \mathrm{~Pb}$ as a function of the encrgy in the laboratory system. The full line corresponds to the adiabatic coupling to all the rotational states, the short-dashed lines are the calculations considering only the ground state orientation and the long-dashed line is a one-channel calculation including only the monopole part of the nuclear potential. A calculation including only the monopole potential will give zero analyzing powers. 
barrier the analyzing powers $T_{20}(\sigma)$ (fig. 3c) are large because the fusion happens most likely when $M=\frac{3}{2}$. Above the barrier $T_{20}(\sigma)$ decreases. For energies well above the barricr it changes sign, which is a consequence of tidal symmetry [8]. The effect of the coupling is to decrease the analyzing power below the barrier (cf. ref. [4]), because it increases relatively more the cross sections for $M=\frac{1}{2}$. The reorientation and coupling values of $T_{20}(\sigma)$ around and above the barrier are very similar, indicating that $T_{20}(\sigma)$ depends only on the magnitude of the quadrupole form factor, and not on the dynamics of the reaction. The values of $T_{20}(J(J+1))$ (fig. 3d) are maximum at energies just above the barrier, and change sign below the barrier.

To summarize, we have presented a model to describe fusion of polarized deformed heavy ions that exhibits the increase in sub-barrier fusion, the enhanccment of $J$-moments above the barrier and large polarization dependence in the fusion cross sections. The first two phenomena are "dynamic" effects, strongly dependent on the moment of inertia of the deformed nucleus. The third phenomenon is a "static" effect, determined by the deformation of the nucleus. Our calculations imply that the analyzing power for fusion above the Coulomb barrier gives a direct measurement of the deformation of the potential that is fairly independent on the reaction mechanism. The effect of coupling produces an increase in sub-barrier fusion that is correlated with an incrcase in the $J$-moments above the barricr. Both effects arc generated by the splitting of the barriers. This correlation may be altered when the reaction mechanisms exhibit an explicit $L$-dependence or energy dependence. This would be the case when transfer channels or neck formation are rclevant.

We can conclude that the performance of experiments in which the fusion cross sections and the $J$ moments of deformed polarized nuclei are measured will be very useful to test our understanding of the reaction mechanisms that lead to fusion. If the coupling to inelastic states is the dominant fcature, one should expect important and correlated increases, with respect to the monopole calculations, in the subbarrier fusion cross sections and in the $J$-moments just above the barrier, that could be calculatcd from the deformation of the potential extracted from the analyzing powers. If this correlation is not found in the experimental data, it could indicate that other phenomena, such as transfer or neck-formation, play an important role.

This work has been partially supported by a grant of Fundación Cámara, the project DGICyT PB890636 and the Accion Integrada HB-196. The authors wish to acknowledge fruitful discussions with M.A. Nagarajan.

\section{References}

[1] M.A. Nagarajan, ed., Proc. Workshop on Hcavy ion collisions at energies near the Coulomb barricr (Daresbury Laboratory, England, July 1990), Institute of Physics Conference Series Number 110 (IOP Publishers, Bristol, $1991)$.

[2] S. Gil et al., Proc. XII Workshop on Nuclear physics (Iguazu Falls, Argentina, 1989) (World Scientific, Singaporc, 1990) p. 19.

[3] R. Vandenbosch, Proc. Workshop on Heavy ion collisions at energies near the Coulomb barrier (Daresbury Laboratory, England, July 1990), ed. M.A. Nagarajan, Institute of Physics Conference Series Number 110 (IOP Publishers, Bristol, 1991) p. 269.

[4] R. Butsch et al., Phys. Rev. C 36 (1987) 1351

[5] H.H. Wolter, Proc. Workshop on Heavy ion collisions at energies near the Coulomb barrier (Daresbury Laboratory, England, July 1990), ed. M.A. Nagarajan, Institute of Physics Conference Series Number 110 (IOP Publishers, Bristol, 1991) p. 259.

[6] I.J. Thompson, Proc. Workshop on Heavy ion collisions at energies near the Coulomb barrier (Daresbury Laboratory, England, July 1990), ed. M.A. Nagarajan, Institute of Physics Conference Series Number 110 (IOP Publishers, Bristol, 1991 ) p. 165, p. 217.

[7] J. Gómez-Camacho and R.C. Johnson, J. Phys. G 12 (1986) L235; G 14 (1988) 609.

[8] J. Gómez-Camacho, Phys. Lett. B 185 (1987) 310.

[9] P. Jacobs and U. Smilansky, Phys. Lett. B 127 (1983) 313.

[10] D.M. Brink, Theory of heavy-ion reactions, in: Nuclear physics with heavy ions and mesons, Vol. 1 (Les Houches, France, July-August 1977), eds. R. Balian, M. Rho and G. Ripka (North-Holland, Amsterdam, 1978) p. 1.

[11] P.M. Endt, Nucl. Phys. A 521 (1990) 12.

[12] R.A. Broglia and A. Winther, Heavy ion reactions, Lecture Notes, Vol. 1 (Benjamin, Reading, MA, 1981). 